\title{
Principal Component Analysis and Support Vector Machine on Ice Entrainment Through a Sluice Gate
}

\author{
Naisheng Liang ${ }^{1}$, Youcai Tuo ${ }^{1}$, Yun Deng ${ }^{1}$, and Tianfu $\mathrm{He}^{1}$ \\ ${ }^{1}$ Sichuan University
}

September 25, 2021

\begin{abstract}
The entrainment and accumulation of ice floes in front of the sluice gates are closely related to the water transport efficiency and safe operation of the channel during an ice period. A flume study is carried out for a sluice gate with free outflow. A framework of stacking ensemble models is used to analyze the data, which consists of a two-level structure including the principal component analysis (PCA) and the support vector machine (SVM) algorithms. Based on the mechanism of ice floe accumulation, ten input characteristics of the machine learning (ML) model are selected. The PCA method is used to eliminate redundant information. The first principal component, with a contribution rate of $71.76 \%$, and the second principal component, with a contribution of rate $15.64 \%$, are extracted as the inputs of the SVM model, and the state of the floating ice in front of the gate is used to determine the classification labels. The 5-fold cross-validation method is used to train the model. The training results showed that the Gaussian radial basis functions (RBF) were the optimal kernel function. The performance of the developed model is measured using area under curve (AUC), accuracy (Acc) and F1-score (F1) values as statistical indicators. The results showed that the established PCA-SVM model improves the Bernoulli naive Bayes (Bernoulli NB) classifier and K-nearest neighbors' algorithm (KNN) models. It increasing the AUC value by $11 \%$ and $5 \%$, the Acc value by $16 \%$ and $17 \%$, and the $\mathrm{F} 1$ value by $17 \%$ and $2 \%$, respectively.
\end{abstract}

\section{Prediction of Ice Entrainment Through a Gate}

Naisheng Liang, Youcai Tuo*, Yun Deng, Tianfu He

State Key Laboratory of Hydraulics and Mountain River Engineering, Sichuan University, Chengdu 610065, Sichuan, China

\section{Hosted file}

Main_text.docx available at https://authorea.com/users/437746/articles/539169-principalcomponent-analysis-and-support-vector-machine-on-ice-entrainment-through-a-sluice-gate 
figures/Figure-1/Figure-1-eps-converted-to.pdf 
figures/Figure-2/Figure-2-eps-converted-to.pdf

\section{Hosted file}

Figure_3.eps available at https://authorea.com/users/437746/articles/539169-principalcomponent-analysis-and-support-vector-machine-on-ice-entrainment-through-a-sluice-gate 
figures/Figure-4/Figure-4-eps-converted-to.pdf 

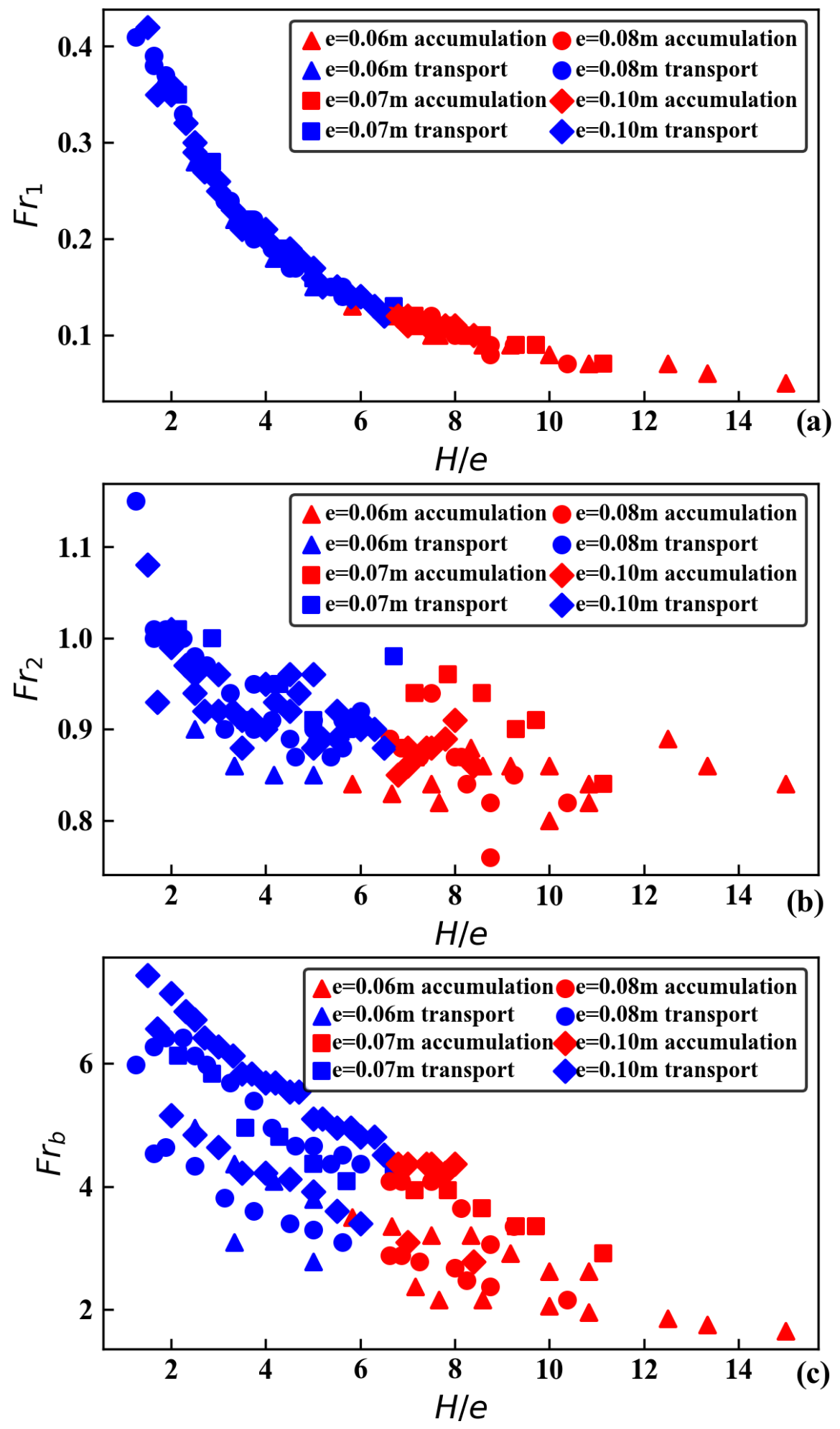

Hosted file

Figure_6.eps available at https://authorea.com/users/437746/articles/539169-principal- 
component-analysis-and-support-vector-machine-on-ice-entrainment-through-a-sluice-gate figures/Figure-7/Figure-7-eps-converted-to.pdf 
figures/Figure-8/Figure-8-eps-converted-to.pdf 
figures/Figure-9/Figure-9-eps-converted-to.pdf

\begin{tabular}{|c|c|c|c|c|c|c|c|c|c|c|c|}
\hline$e$ & 1 & $|0.018|$ & 0.63 & $|0.004|$ & 0.66 & -0.24 & -0.39 & 0.3 & 0.29 & 0.55 & 1.00 \\
\hline$H-$ & -0.018 & 1 & -0.73 & 0.97 & $-0.66 \mid$ & 0.83 & 0.9 & -0.63 & -0.87 & -0.65 & 0.75 \\
\hline$V$ & 0.63 & -0.73 & 1 & -0.72 & 0.97 & -0.83 & -0.9 & 0.77 & 0.9 & 0.87 & 0.50 \\
\hline$V_{e}$ & 0.004 & 0.97 & -0.72 & 1 & $-0.65 \mid$ & 0.9 & 0.87 & -0.59 & -0.91 & -0.64 & 0.25 \\
\hline$V_{s}$ & 0.66 & $-0.66 \mid$ & 0.97 & -0.65 & 1 & -0.77 & $\mathbf{- 0 . 8 7}$ & 0.75 & 0.84 & 0.83 & 000 \\
\hline$H_{1} / H$ & -0.24 & 0.83 & -0.83 & 0.9 & $|-0.77|$ & 1 & 0.83 & -0.79 & -0.98 & -0.74 & \\
\hline H/e & -0.39 & 0.9 & -0.9 & 0.87 & -0.87 & 0.83 & 1 & -0.67 & -0.89 & -0.81 & -0.25 \\
\hline$F r_{1}$ & 0.3 & -0.63 & 0.77 & -0.59 & 0.75 & -0.79 & -0.67 & 1 & 0.81 & 0.68 & -0.50 \\
\hline$F r_{2}$ & 0.29 & -0.87 & 0.9 & -0.91 & 0.84 & -0.98 & -0.89 & 0.81 & 1 & 0.79 & -0.75 \\
\hline$F r_{b}$ & 0.55 & -0.65 & 0.87 & -0.64 & 0.83 & -0.74 & -0.81 & 0.68 & $\mid 0.79$ & 1 & \\
\hline
\end{tabular}




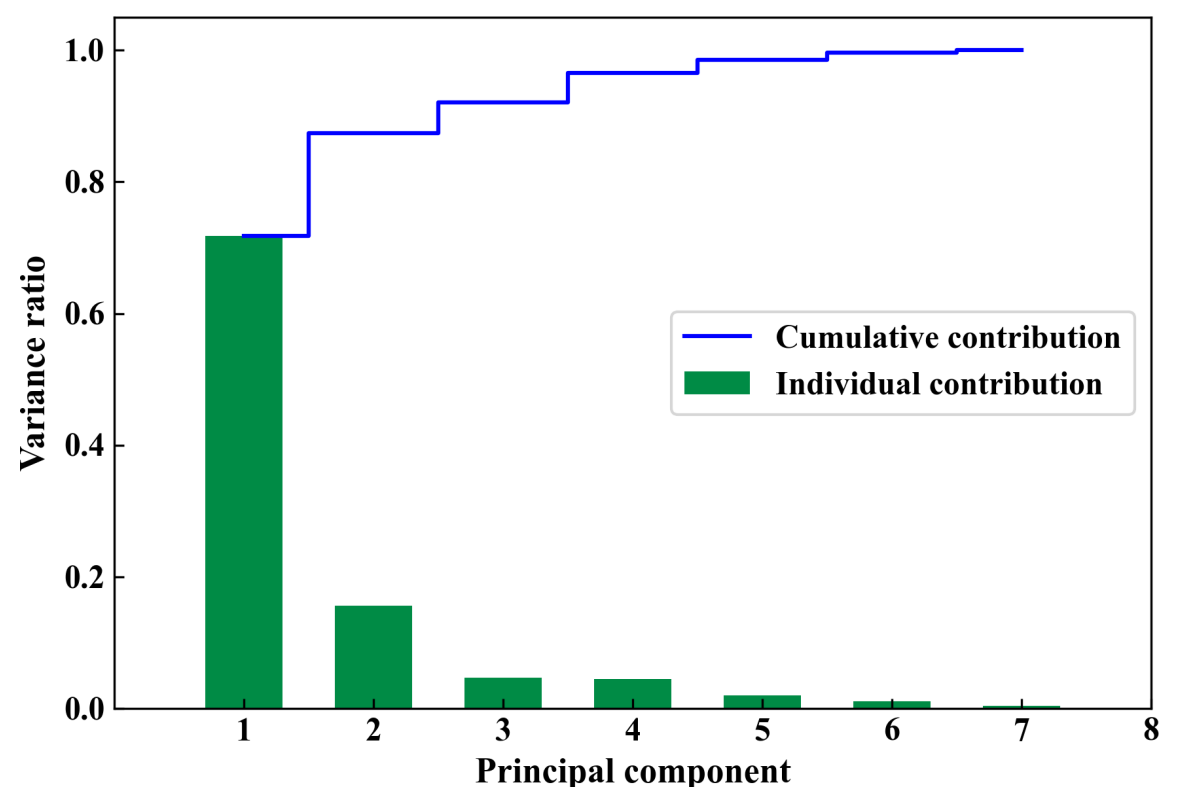



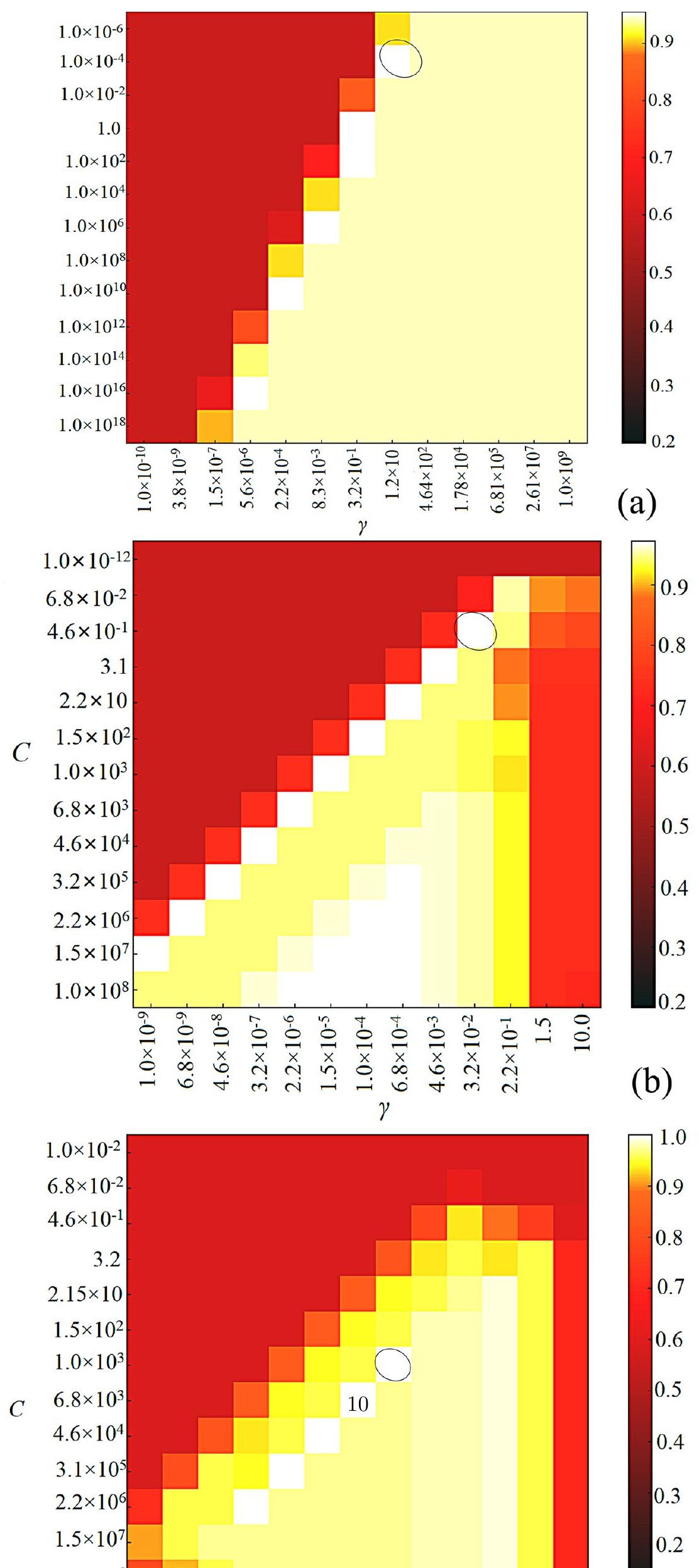

(b) 

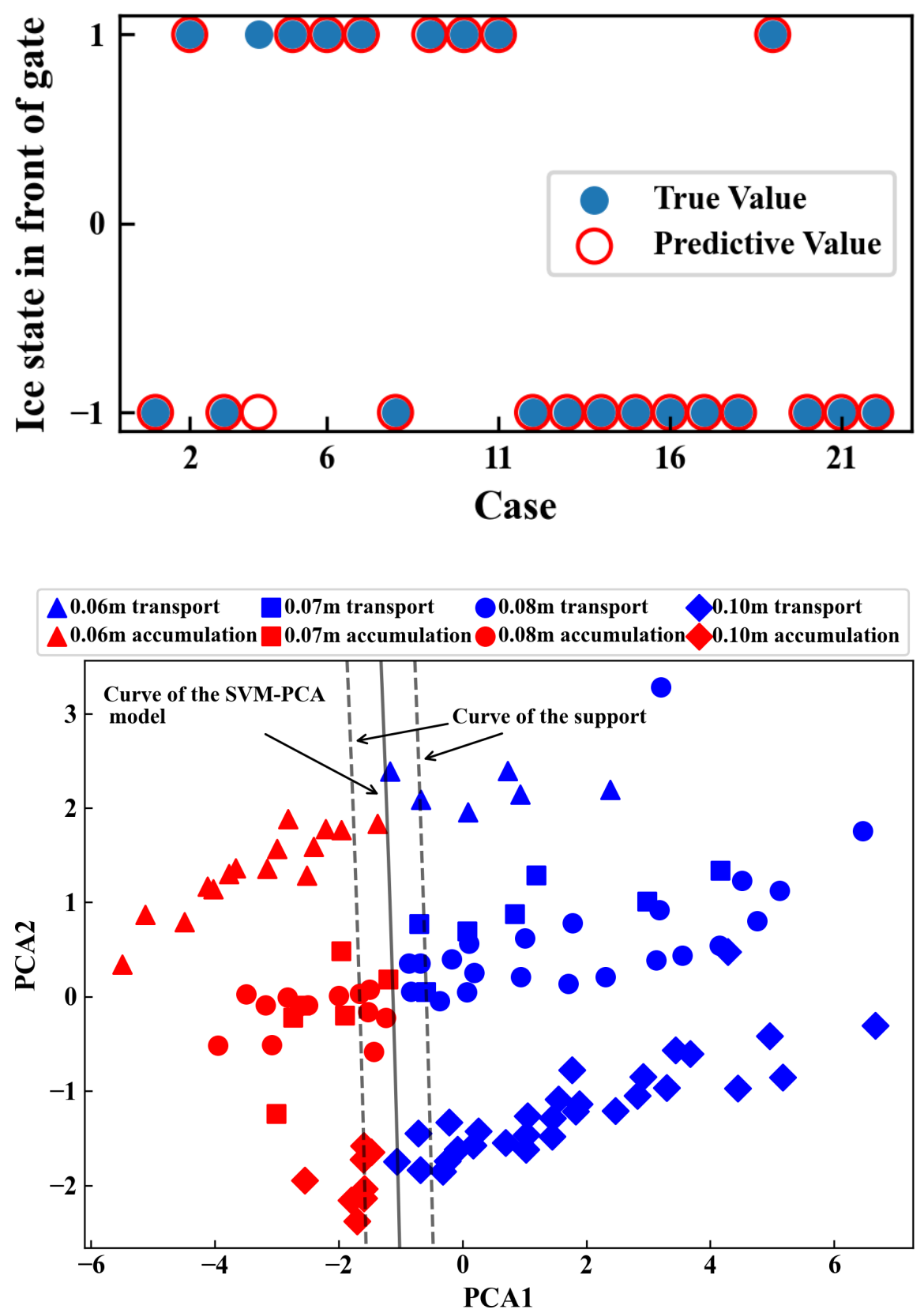

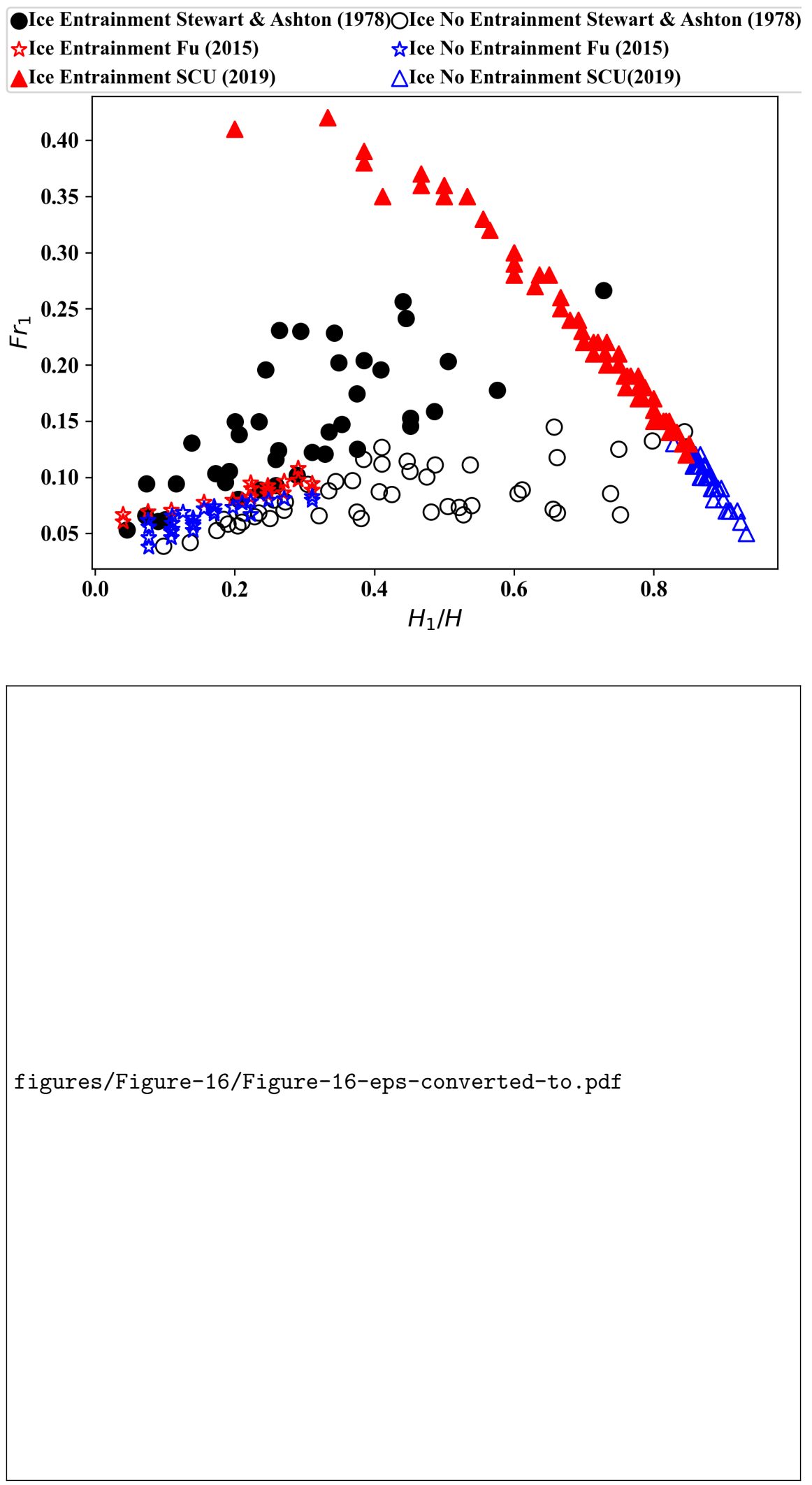

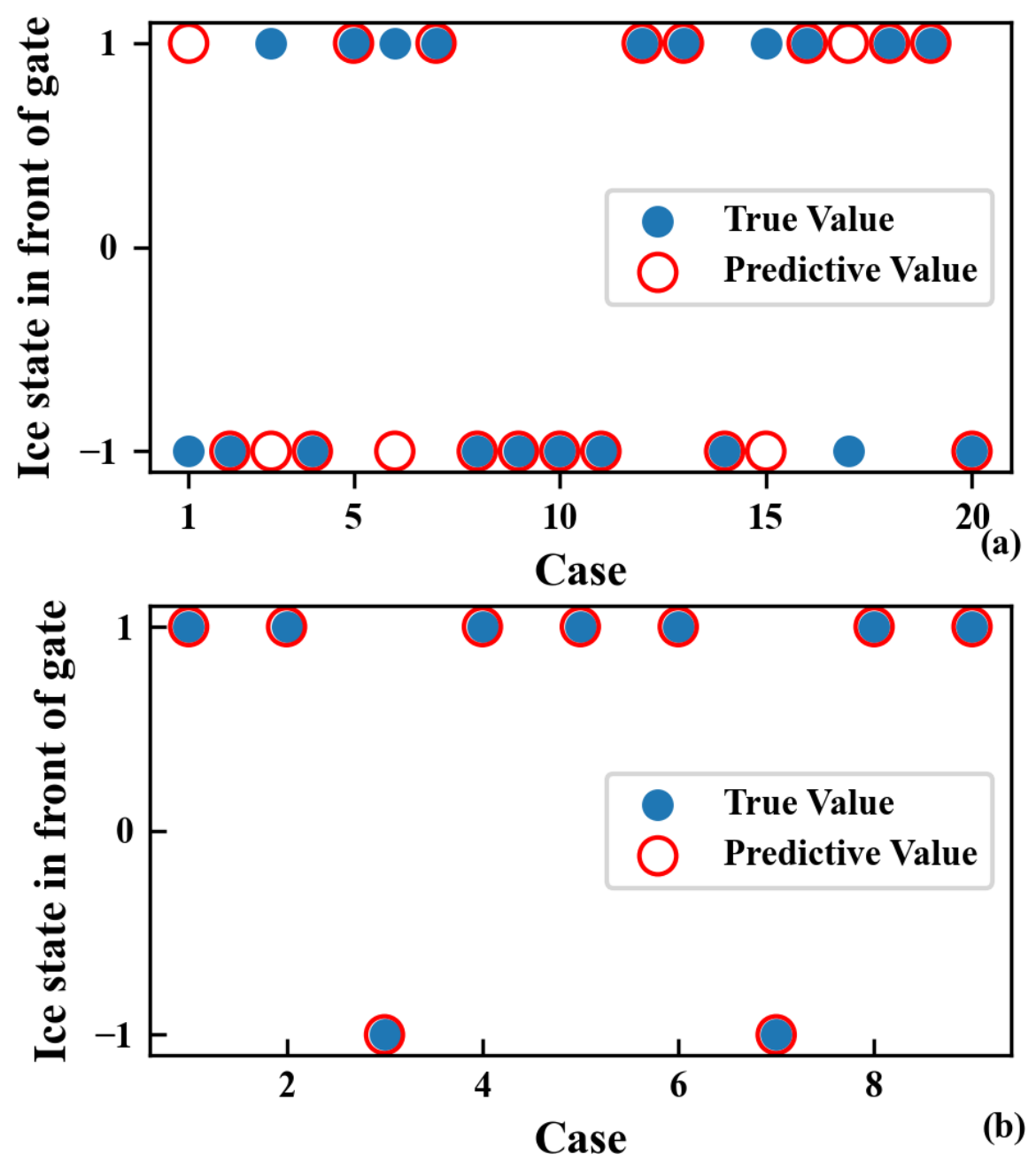